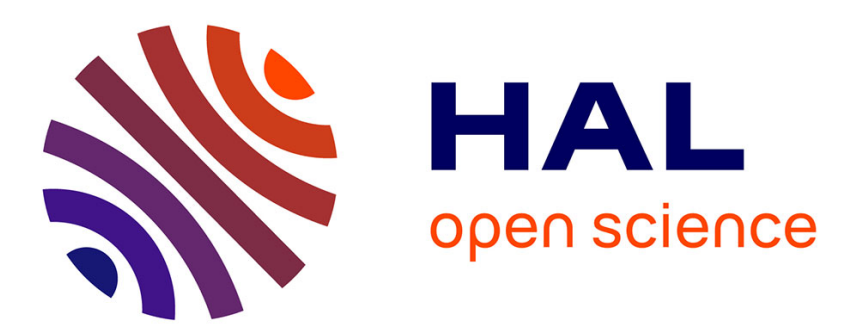

\title{
A Switched Control Method for Networked Control Systems
}

Xu-Guang Li, Arben Cela, Silviu-Iulian Niculescu, Abdellatif Reama

\section{To cite this version:}

Xu-Guang Li, Arben Cela, Silviu-Iulian Niculescu, Abdellatif Reama. A Switched Control Method for Networked Control Systems. 8th IFAC Workshop on Time-Delay Systems, Sep 2009, Sinaia, Romania. pp.61-65, 10.3182/20090901-3-RO-4009.00007 . hal-02291603

\section{HAL Id: hal-02291603 https://hal.science/hal-02291603}

Submitted on 19 Sep 2019

HAL is a multi-disciplinary open access archive for the deposit and dissemination of scientific research documents, whether they are published or not. The documents may come from teaching and research institutions in France or abroad, or from public or private research centers.
L'archive ouverte pluridisciplinaire HAL, est destinée au dépôt et à la diffusion de documents scientifiques de niveau recherche, publiés ou non, émanant des établissements d'enseignement et de recherche français ou étrangers, des laboratoires publics ou privés. 


\title{
A Switched Control Method for Networked Control Systems
}

\author{
Xu-Guang Li ${ }^{* * *}$ Arben Çela* Silviu-Iulian Niculescu ${ }^{* *}$ \\ Abdellatif Reama* \\ * UPE, ESIEE Paris, Department of Embedded Systems, 93162, \\ Noisy-Le-Grand, France(Email: masdanlee@163.com,lxu@esiee.fr; \\ a.cela@esiee.fr; a.reama@esiee.fr) \\ ** Laboratoire des Signaux et Systèmes (L2S, UMR CNRS 8506), \\ CNRS-Supélec, 91192, Gif-Sur-Yvette, France (Email: li@lss.supelec.fr; \\ Silviu.Niculescu@lss.supelec.fr)

\begin{abstract}
The performance of networked control systems based on a switched control law is studied. The control law is switched between sampled-data feedback and zero control, the switch instant of which is determined by a switching parameter. The objective of the paper is to analyze 1) under what condition the switched control law can lead to better performance than the standard one and 2) how to select the switching parameter if the condition is satisfied. Numerical examples show the effectiveness of the proposed results.
\end{abstract}

Keywords: Networked control systems (NCSs), switched systems, optimal control.

\section{INTRODUCTION}

Employing a control network will bring various advantages, such as increased system reliability, ease of diagnosis and maintenance. The Networked Control System (NCS) has been an active research topic during the past several decades. A detailed introduction to NCSs can be found in Hespanha, Naghshtabrizi, and Xu (2007), HristuVarsakelis and Levin (2005), and Zhang, Branicky, and Phillips (2001).

In NCSs, zero-order-hold ( $\mathrm{ZOH})$ devices are widely used since the sampling rate can not be infinitely large. As a result, between two consecutive sampling instants, $k T \leq$ $t<(k+1) T$, the controller can only use the sampled data $x(k T)$. Thus, a linear feedback control law $u(t)=K x(k T)$ for $k T \leq t<(k+1) T$. This kind of control law, which will be called the standard control law in this paper, can be seen almost everywhere in the studies of NCSs, see e.g. Fridman, Seuret, and Richard (2004), Mirkin (2007), Fujioka (2008).

In this paper, we will employ an easily-implemented switched control law instead of the standard one. The basic idea is that when $k T \leq t<k T+a$, let $u(t)=K x(k T)$; while when $k T+a \leq t<(k+1) T, u(t)=0$, where $a$ $(0 \leq a \leq T)$ is the switching parameter to be designed.

When using the standard control law, the closed-loop system is equivalent to an input-delayed system with delay $\tau(t)=t-k T$, as analyzed in Fridman, Seuret, and Richard (2004). Due to the effect of delay, we believe that in a certain time interval the open-loop dynamic (i.e. $u(t)=0$ ) is more favorable for the system to "converge". This is the reason we employ the new control law. Here, we give a motivating example. Consider the system with matrices below equation (6) and with sampling $T=1.7$. The system is asymptotically stable and has a Lyapunov function $V(t)=x^{T}(t) P x(t)$ with $P=[4.035 .09 ; 5.09$ 13.49]. The trajectory of $V(t)$ is shown in Fig. 1. It is seen that at sampling instants, $V(t)$ monotonously decreases. However, between sampling instants, $V(t)$ first decreases and then increases. For this system, as time increases between two sampling instant, the standard control law gradually loses its stabilizing effect. We then employ a switched control with switching parameter $a=0.94$ and make a simulation for $V(t)$, see the bold curve in Fig. 1. It is clear that the switched control law lead better performance than the standard one.

The switched control law has been used in Sun, Liu, Rees, and Wang (2008), where it is called even-time-driven control. Therein, a Lyapunov-Krasovskii method is used to estimating the decay rate of the closed-loop system and diverge rate of the open-loop system. The stability is guaranteed if the system is on average convergent over the whole sampling period. The method proposed in this paper is different with that used in Sun, Liu, Rees, and Wang (2008).

In this paper, we will address the following problems: Under what condition, the standard control law is better and under what condition, the new control law is better? (Through the motivating examples given later, we will see that the new control law does not always lead to better performance) If the new control law can lead to better performance, how should we choose a switching time? Optimal control for networked control systems has been widely considered, see e.g., Ben Gaid, Çela, and Hamam (2006) and Chen and Francis (1991). In this paper, we will use a different method, with the existing ones, to design a switching parameter. 


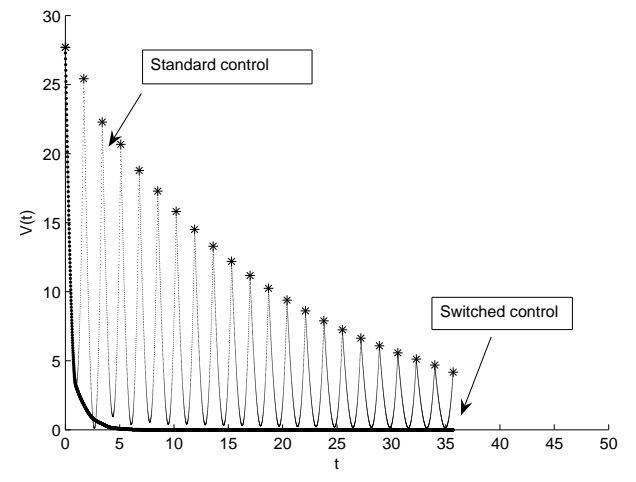

Fig. 1. A motivating example

The analysis in this paper is based on testing the derivative of the performance with respect to the switching parameter. Given a performance index $V=\sum_{i=1}^{N} x^{T}(i T) Q x(i T)$, we can have $d V / d a=x^{T}(0) \Theta^{\prime}(a) x(0)$, as will be shown later in the text. Thus, in an interval where $\Theta^{\prime}(a)$ is negative-definite (positive-definite), the system performance monotonously decreases (increases) with respect to the switching parameter $a$. If in an interval where $\Theta^{\prime}(a)$ is indefinite (neither negative-semidefinite or positivesemidefinite), there does not exist an optimal switching parameter. In this case, the optimal switching parameter varies with respect to different initial condition. According to the property (negative-definite, positive-definite or indefinite) of $\Theta^{\prime}(a)$, we can tell if the switched control law can lead to better performance than the standard one and how to choose the switching parameter. Numerical examples show the effectiveness of the proposed method.

The remaining paper is organized as follows. In Section 2, the optimization problem of a networked control system with the switched control is formulated and some motivating examples are given. In Section 3, main results of the paper are proposed. Numerical examples are presented in Section 4, and some concluding remarks end the paper.

\section{PROBLEM FORMULATION AND MOTIVATING EXAMPLES}

Consider a networked control system with a switched control law

$$
\begin{gathered}
\dot{x}(t)=A x(t)+B u(t) \\
u(t)=\left\{\begin{array}{l}
K x(k T), k T \leq t<k T+a \\
0, k T+a \leq t<(k+1) T
\end{array}\right.
\end{gathered}
$$

where $A, B, K$ are $n \times n, n \times m, m \times n$ real matrices, $T$ is the sampling period, $k$ are nonnegative integers and $a$ is the switching parameter satisfying $0 \leq a \leq T$.

It is assumed that matrix $A$ is not Hurwitz and $K$ is designed such that $A+B K$ is Hurwitz, without considering the effect of network.

The standard control law is given by

$$
u(t)=K x(k T), k T \leq t<(k+1) T
$$

Table 1. Performance for motivating example 1

\begin{tabular}{|c|c|c|c|}
\hline & $(1,1)^{T}$ & $(1,-1)^{T}$ & $(1,2)^{T}$ \\
\hline 0.3 & 34.5178 & 1.3156 & 16.0483 \\
\hline 0.5 & 16.1623 & 1.0396 & 13.4389 \\
\hline 0.7 & 8.0496 & 0.8277 & 11.2502 \\
\hline 0.9 & 5.2061 & 0.6687 & 9.4186 \\
\hline
\end{tabular}

Under the standard control law (3), which is implemented by the zero-order-hold $(\mathrm{ZOH})$ devices, the control value is fixed as $K x(k T)$ until the new sampled data $x((k+1) T)$ arrives.

The controller (2) used in this paper has two switched control status: between $k T \leq t<k T+a$, it uses the value $K x(k T)$, as the standard controller does; however between $k T+a \leq t<(k+1) T$, it uses the value 0. System (1) under controller (2) thus can be expressed in the following switched system:

$$
\dot{x}(t)=\left\{\begin{array}{l}
A x(t)+B K x(k T), k T \leq t<k T+a \\
A x(t), k T+a \leq t<(k+1) T
\end{array}\right.
$$

We choose control law (2) instead of the standard one (3) based on the following analysis:

When employing the standard control law (3), the networked control system can be equivalently written as

$$
\dot{x}(t)=A x(t)+B K x(t-\tau(t)), k T \leq t<(k+1) T
$$

where $\tau(t)=t-k T, k T \leq t<(k+1) T$.

Between two sampling instants, the delay $\tau(t)$ increases with respect to time. Therefore, it is possible that in some time interval the open-loop dynamic $\dot{x}(t)=A x(t)$ is more favorable than the closed-loop dynamic $\dot{x}(t)=A x(t)+$ $B K x(k T)$ for the system performance, due to the effect of delay. If this is true, after an appropriate switching instant $k T+a$, we set the control values as 0 (which is easy to implement in practice) instead of the sampleddata feedback such that an improved performance can be obtained.

In this paper, we choose the following performance index:

$$
V=\sum_{i=1}^{N} x^{T}(i T) Q x(i T)
$$

And the system matrices are given by

$$
\begin{gathered}
A=\left(\begin{array}{cc}
0 & 1 \\
0 & -0.1
\end{array}\right), B=\left(\begin{array}{c}
0 \\
0.1
\end{array}\right) \\
K=(-3.75-11.5), Q=\left(\begin{array}{cc}
4.03 & 5.09 \\
5.09 & 13.49
\end{array}\right) .
\end{gathered}
$$

Two motivating examples are given before the end of this section.

Motivating example 1: When $T=0.9$ and $N=2$. We choose four switching parameters $0.3,0.5,0.7,0.9$ and three initial state $(1,1)^{T},(1,-1)^{T},(1,2)^{T}$. We compute the performance under different switching parameters and initial conditions, see Table.1 for the results.

From the table, we can see that the optimal performance is always obtained when $a=T=0.9$. That is to say, the 
Table 2. Performance for motivating example 2

\begin{tabular}{|c|c|c|c|}
\hline & $(1,1)^{T}$ & $(1,-1)^{T}$ & $(1,2)^{T}$ \\
\hline 0.6 & 12.8753 & 2.5601 & 33.7183 \\
\hline 0.9 & 4.4923 & 0.5462 & 8.3129 \\
\hline 1.0 & 4.1121 & 0.5059 & 7.2336 \\
\hline 1.2 & 6.0208 & 0.9400 & 12.9831 \\
\hline
\end{tabular}

control law (2) can not lead to better performance than the standard control law (3), for this example.

Motivating example 2: When $T=1.2$ and $N=100$. We choose four switching parameters $0.6,0.9,1.0,1.2$ and three initial state $(1,1)^{T},(1,-1)^{T},(1,2)^{T}$. The computed performance are listed in Table.2.

From the table, we can see that if switched at some but not arbitrary instant, the control law (2) can lead to better performance than the standard control law (3).

Now, one may naturally have two questions on the switched control law (2): 1) Under what condition, we can obtain better performance by using the switched control law (2) than using the standard one (3)? How should we choose an appropriate switching parameter to obtain an improved performance? The objective of this paper is to answer these two questions. Two emphasize the effect of switching parameter, we do not take the transmission delay into account. We only consider a simple form of performance index here. The method of this paper can be further extended to the cases with transmission delay and with other performance indices.

The stability analysis, not the purpose of this paper, for NCSs based on the switched control (2) is reported in Li, Cela, Niculescu, and Reama (2009b). It is shown that larger stability interval can be obtained by employing the switched control (2). For the system considered in this paper, it the sampling period is constant, the stability interval is $T \in[0,1.724)$ by the standard control (3). By the switched control (2), the stability interval is $T \in$ $[a,+\infty)$, if $a \in(0,0.534)$. If the sampling periods are time-varying, the stability interval is $T_{k} \in[0,1.72]$ by the standard control (3). By the switched control (2), the stability interval is $T_{k} \in[0,10.25]$.

\section{MAIN RESULTS}

The system described by (1) and (2), with the initial state $x(0)$ has the following response:

$$
\begin{aligned}
& x(t)= \\
& \left\{\begin{array}{l}
\Phi(t-k T, t-k T) \Phi^{k}(a, T) x(0), k T \leq t<k T+a \\
\Phi(a, t-k T) \Phi^{k}(a, T) x(0), k T+a \leq t<(k+1) T
\end{array}\right.
\end{aligned}
$$

where

$$
\Phi(a, T)=e^{A(T-a)}\left(e^{A a}+\int_{0}^{a} e^{A \theta} d \theta B K\right)
$$

And the discrete-time expression of the system is

$$
x((k+1) T)=\Phi(a, T) x(k T)
$$

Remark 1. It is easy to see that when $a=T$, the switched control law (2) is equivalent to the standard one (3).
Given a sampling period $T$, the performance index (6) can be rewritten as

$$
V(a)=x^{T}(0) \Theta(a) x(0)
$$

where

$$
\Theta(a)=\sum_{i=1}^{N}\left(\Phi^{i}(a, T)\right)^{T} Q \Phi^{i}(a, T)
$$

It can be seen that for given $x(0), T$ and $N$, the system performance depends on the switching parameter $a$. Some results are given below to show the relationship between the performance $V(a)$ and the switching parameter $a$. Throughout this paper, $A>0(A<0)$ denotes that matrix $A$ is positive-definite (negative-definite).

Theorem 1. If there exists an interval $\left[\underline{a}_{-}, \bar{a}_{-}\right]$such that $\Theta^{\prime}(a)<0$ for $a \in\left[\underline{a}_{-}, \bar{a}_{-}\right]$

where

$$
\begin{gathered}
\Theta^{\prime}(a)=\sum_{i=1}^{N}\left(d \frac{\Phi^{i}(a, T)^{T}}{d a} Q \Phi^{i}(a, T)+\Phi^{i}(a, T)^{T} Q d \frac{\Phi^{i}(a, T)}{d a}\right), \\
d \frac{\Phi(a, T)}{d a}=\left(e^{A T}-A e^{A(T-a)} \int_{0}^{a} e^{A \theta} d \theta\right) B K,
\end{gathered}
$$

it follows that $V\left(a_{1}\right)>V\left(a_{2}\right)$ for any $a_{1}, a_{2}$ with $\underline{a}_{-} \leq$ $a_{1}<a_{2} \leq \bar{a}_{-}$and any initial state $x(0)$.

If there exists an interval $\left[\underline{a}_{+}, \bar{a}_{+}\right]$such that $\Theta^{\prime}(a)>0$ for $a \in\left[\underline{a}_{+}, \bar{a}_{+}\right]$, it follows that $V\left(a_{1}\right)<V\left(a_{2}\right)$ for any $a_{1}, a_{2}$ with $\underline{a}_{+} \leq a_{1}<a_{2} \leq \bar{a}_{+}$and any initial state $x(0)$.

Proof. One can see that $d \frac{V(a)}{d a}=x^{T}(0) \Theta^{\prime}(a) x(0)$. For any initial state $x(0)$, we have $V(a)$ decreases (increases) as $a$ increases if $\Theta^{\prime}(a)<0\left(\Theta^{\prime}(a)>0\right)$. The proof is complete.

Corollary 1. If $\Theta^{\prime}(a)<0$ for $a \in[0, T]$, the control law (2) can not lead to better performance than the control law (3).

Proof. If $\Theta^{\prime}(a)<0$ for $a \in[0, T]$, we always have the minimal performance when $a=T$, which is exactly the standard control law (3).

Corollary 2. If there exists an interval $\left[T_{1}, T\right]\left(0 \leq T_{1}<T\right)$ such that $\Theta^{\prime}(a)>0$ for $a \in\left[T_{1}, T\right]$, the control law (2) can lead to better performance than the control law (3) under any initial state.

Proof. One can see that there exist $a \in\left[T_{1}, T\right)$ such that $V(a)<V(T)$ under any initial state, if $\Theta^{\prime}(a)>0$ for $a \in\left[T_{1}, T\right]$.

Theorem 2. If there exists an interval $\left[\underline{a}_{0}, \bar{a}_{0}\right]$ such that $\Theta^{\prime}(a)$ is indefinite (neither negative-semidefinite nor positivesemidefinite) for $a \in\left[a_{0}, \bar{a}_{0}\right]$, there does not exist an optimal switching parameter, say $a^{*}$, in this interval such that $V\left(a^{*}\right)<V(a)$ for $a \neq a^{*} \in\left[\underline{a}_{0}, \bar{a}_{0}\right]$ under any initial condition.

Proof. Assume that there is an optimal switching parameter $a^{*}$. For a positive $a^{\#}$, which is sufficiently close to $a^{*}$, we have $\Theta\left(a^{*}\right)-\Theta\left(a^{\#}\right)$ is indefinite since in $\left[\underline{a}_{0}, \bar{a}_{0}\right], \Theta^{\prime}(a)$ is indefinite. Therefore, there exists an initial state $x(0)$ 
satisfying $V\left(a^{*}\right)-V\left(a^{\#}\right)>0$. The result is proved by contradiction.

Corollary 3. If there exists an interval $a \in\left[T_{2}, T\right](0 \leq$ $\left.T_{2}<T\right)$ where $\Theta^{\prime}(a)$ is indefinite, the control law (2) can lead to better performance than the control law (3) under some (not all) initial state.

This result follows straightforwardly from Theorem 2 .

Remark 2. Sometimes, the optimal switching parameter does not exist. However, we can have an indefinite interval such that for any initial state the optimal switching parameter lie in this interval. We call this interval quasioptimal interval in this paper. This will be seen in the numerical example section

The eigenvalues of $\Theta^{\prime}(a)$ are the roots of the following characteristic equation

$$
C E=\operatorname{det}\left(\lambda I-\Theta^{\prime}(a)\right)
$$

The highest degree of $C E$ is fixed. Thus, as analyzed in $\mathrm{Li}$, Çela, Niculescu, and Reama (2009a), the eigenvalues of $\Theta^{\prime}(a)$ are continuous with respect to $a$ and the parameter sweeping technique is effective to check the property (negative-definite, positive-definite or indefinite) of $\Theta^{\prime}(a)$. The parameter sweeping technique can be easily realized by standard computing software such as MATLAB. It is worth to note that the parameter sweeping technique has been widely used in the stability analysis, see, e.g., Fazelinia, Sipahi, and Olgac (2007) and Gu, Niculescu, and Chen (2005).

\section{NUMERICAL EXAMPLES}

In this section, some numerical examples are given to illustrate the proposed method in this paper.

We choose the system (1) under the switched control law (2) with matrices $A, B, K, Q$ given in section 2 .

Example 1: When $T=0.9$ and $N=2$. By parametersweeping, we find for $a \in[0,0.9], \Theta^{\prime}(a)<0$. According to Theorem 1, we know the performance monotonously decreases with respect $a$ and the optimal performance is always obtained at $a=T=0.9$. In other words, the control law (2) can not lead to better performance than the standard control law (3), according to Corollary 1 . We choose three initial conditions $(1,1)^{T},(1,-1)^{T}$, and $(1,2)^{T}$ for simulation, see Fig. 2. The simulations coincide with the proposed results in this paper.

Example 2: When $T=1.2$ and $N=100$.

By parameter-sweeping, we have $\Theta^{\prime}(a)<0$ for $a \in$ $[0,0.810)$ and $\Theta^{\prime}(a)>0$ for $a \in(1.003,1.2]$. Therefore, the quasi-optimal switching parameters should lie in the interval $[0.810,1.003]$ (the indefinite interval). For any initial state, the optimal performance will be obtained in the interval [0.810, 1.003].

For this example, we choose three initial state $(1,1)^{T}$, $(-2,1)^{T}$, and $(3,-1)^{T}$. And for each simulation, a closeup graph is added, see Figures 2-7. For initial state $(1,1)^{T}$, $(-2,1)^{T}$, and $(3,-1)^{T}$, we have the optimal switching parameter $0.99,0.88$, and 0.84 , respectively.

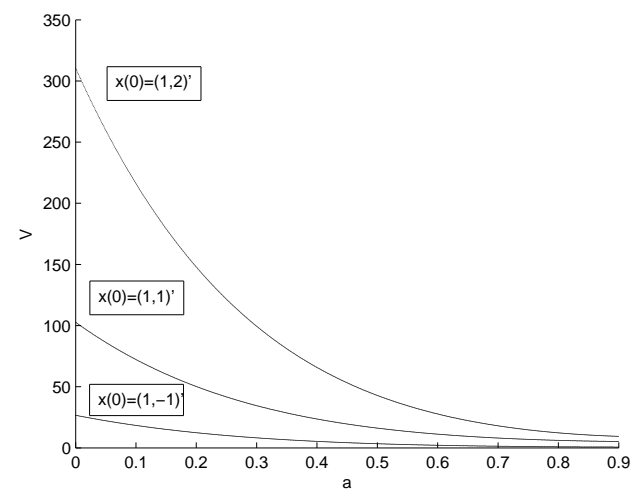

Fig. 2. Simulation for example 1

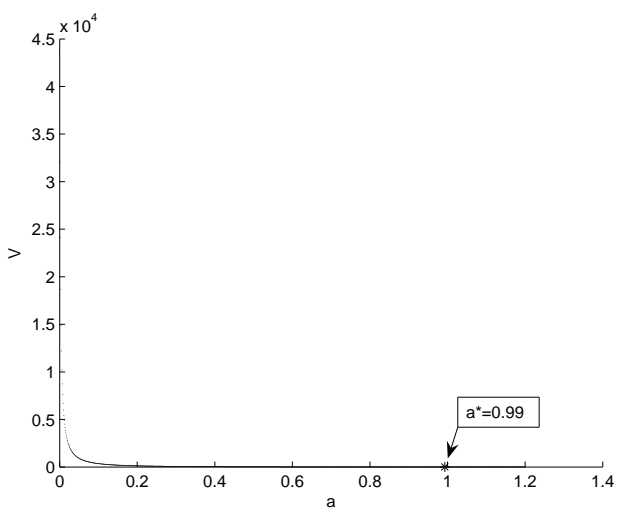

Fig. 3. Simulation for example 2 with $x_{0}=(1,1)^{T}$

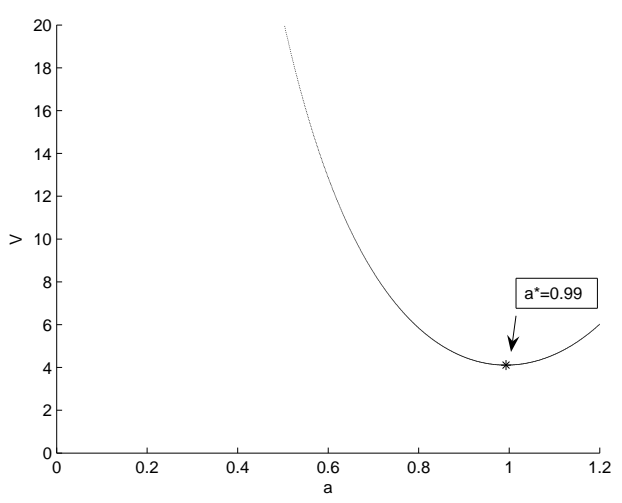

Fig. 4. Simulation for example 2 with $x_{0}=(1,1)^{T}$ (closeup)

Through the simulation, we can see the performance monotonously decreases as $a$ increases in the interval $[0,0.810)$, while monotonously increases as $a$ increases in the interval $(1.003,1.2]$. The optimal switching parameter varies depending on the initial state, however it must be inside $[0.810,1.003]$. For this example, we can obtain improved performance when employing the switched control law (2) than employing the standard control law (3).

\section{CONCLUSION}

The performance of networked control systems based on a switched control law is considered. The relationship 


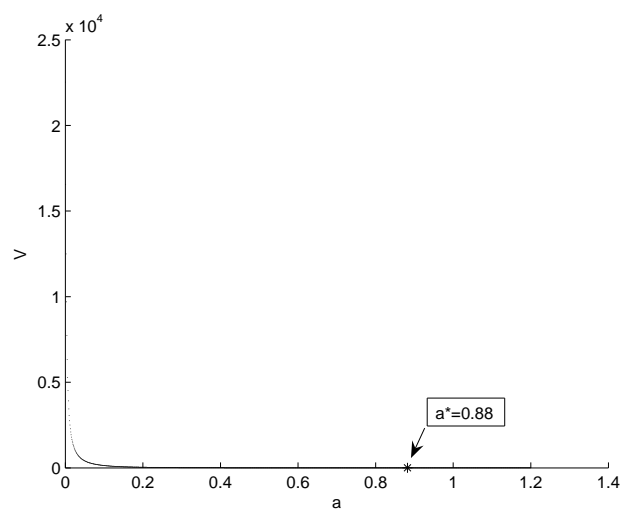

Fig. 5. Simulation for example 2 with $x_{0}=(-2,1)^{T}$

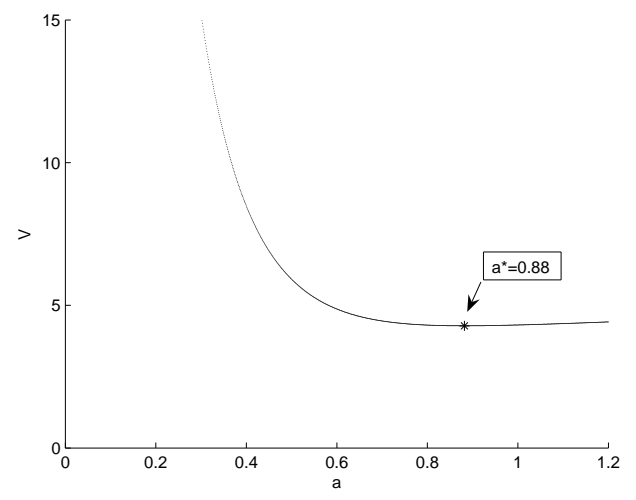

Fig. 6. Simulation for example 2 with $x_{0}=(-2,1)^{T}$ (closeup)

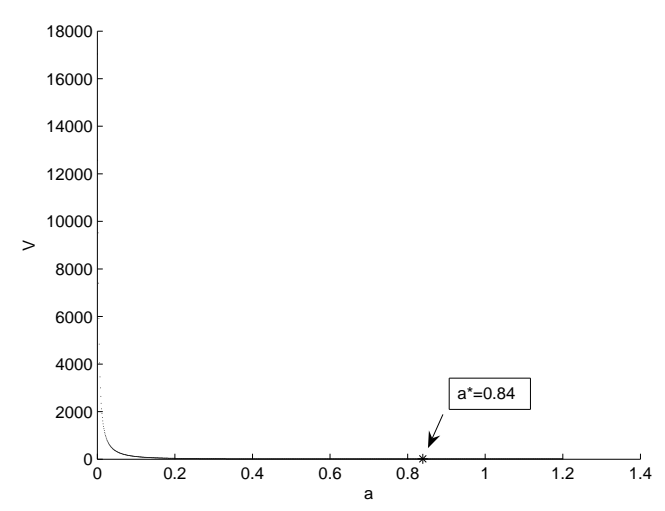

Fig. 7. Simulation for example 2 with $x_{0}=(3,-1)^{T}$

between the switching parameter and the system performance is analyzed. The method is based on testing the property (negative, positive or in-definite) of the performance derivative with respect to the switching parameter. As a result, we know if the switched control law can lead to better performance than the standard one and how to select a switching parameter.

\section{REFERENCES}

Ben Gaid, M.E.M., Çela, A., and Hamam, Y., (2006). Optimal integrated control and scheduling of networked control systems with communication constraints: appli-

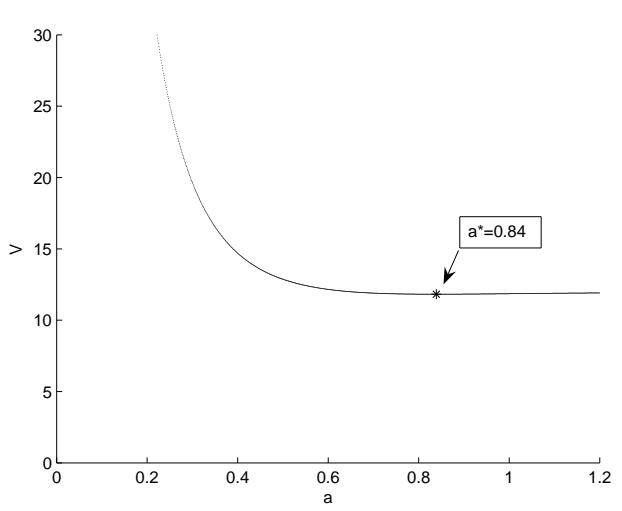

Fig. 8. Simulation for example 2 with $x_{0}=(3,-1)^{T}$ (closeup)

cation to a car suspension system. IEEE Transactions on Control Systems Technology, 14(4), 776-787.

Chen, T., Francis, B.A. (1991). $H_{2}$-optimal sampleddata control. IEEE Transactions on Automatic Control, 36(4), 387-397.

Fazelinia, H., Sipahi, R., and Olgac, N., (2007). Stability robustness analysis of multiple time-delayed systems using "Building Block" concept. IEEE Transactions on Automatic Control, 52(5), 799-810.

Fridman, E., Seuret, A., and Richard, J.-P.,(2004). Robust sampled-data stabilization of linear systems: an input delay approach. Automatica, 40(8), 1441-1446.

Fujioka, H.,(2008). Stability analysis for a class of networked/embedded control systems: A discrete-time approach. American Control Conference Seattle, Washington, USA, pp.4997-5002.

Gu, K., Niculescu, S.-I., and Chen, J.,(2005). On stability of crossing curves for general systems with two delays. Journal of Mathematical Analysis and Applications, 311(1), 231-253.

Hespanha, J.P., Naghshtabrizi, P., and Xu, Y.,(2007). A survey of recent results in networked control systems. Proceedings of the IEEE, 95(1), 138-162.

Hristu-Varsakelis, D. and Levine, W.S. Eds.,(2005). Handbook of Networked and Embedded Control Systems. Boston: Birkhäuser.

Li,X.-G., Çela, A., Niculescu, S.-I., and Reama, A.,(2009a). Some remarks on the stability of networkedcontrol systems with periodic scheduling. To appear in European Control Conference, Budapest, Hungary.

Li,X.-G., Çela, A., Niculescu, S.-I., and Reama, A.,(2009b). Stability analysis of networked control systems based on a switched control. To submit.

Mirkin, L.,(2007). Some remarks on the use of timevarying delay to model sample-and-hold circuits. IEEE Transactions on Automatic Control, 52(6), 1109-1112.

Sun, X.-M., Liu, G.-P., Rees, D., and Wang, W. (2008). A novel method of stability analysis for networked control systems. Proceedings of the 17th World Congress, IFAC, Seoul, Korea, 4852-4856.

Zhang, W., Branicky, M.S., and Phillips, S.M.,(2001). Stability of networked control systems. IEEE Control Systems Magazine, 21(1), 84-99. 